\title{
Impending Doom: Antibiotic Exposure and Bacterial Gene Expression
}

\author{
Timothy Palzkill \\ Department of Molecular Virology and Microbiology, Baylor College of Medicine, Houston, Texas 77030, USA
}

\begin{abstract}
$\mathbf{T}$ he discovery of antibiotics ranks as one of the most significant medical achievements of the past century: Antibiotics have greatly attenuated the severity and lethality of bacterial infections. Because antibiotics have been so widely successful, they have been extensively used. This has resulted in strong selective pressure on bacteria that, in turn, has led to the rapid and widespread evolution of antibiotic resistance. Antibiotic resistance now poses a serious threat to the therapy of certain bacterial infections, and new approaches are needed for the development of the next generation of antibiotics. Functional genomics technologies to examine RNA and protein expression levels on a genomewide scale may prove to be important tools for drug discovery research.

In this issue Gmuender et al. (2000) use a functional genomics approach to examine the changes in gene expression that occur in the human respiratory pathogen Haemophilus influenzae upon exposure to the antibiotics ciprofloxacin and novobiocin. Although both of these drugs inhibit bacterial DNA gyrase, they do so by different mechanisms. Novobiocin is a coumarin antibiotic that binds to the ATP binding site of the B subunit of gyrase and inhibits the supercoiling activity of the enzyme. Because transcription is known to be sensitive to the state of supercoiling, novobiocin may affect the transcription of many genes. In contrast, ciprofloxacin is a quinolone that binds to the A subunit of gyrase and interrupts the DNA cleavage and resealing activity of the enzyme. The failure to seal doublestrand breaks
\end{abstract}

E-MAIL timothyp@bcm.tmc.edu; FAX (713) 7987375.

Article and publication are at www.genome.org/cgi/ doi/10.1101/gr.170901. results in DNA damage and quinolones are known to induce DNA repair systems (Piddock et al. 1990). Thus, the two drugs may elicit different physiological responses despite binding to the same target.

A distinguishing feature of this work is that Gmuender et al. (2000) examine gene expression at the level of transcription with microarrays and translation using two-dimensional polyacrylamide gel electrophoresis. The use of parallel cultures for the transcription and translation studies permits a direct comparison between differential RNA synthesis versus protein synthesis. The authors find that absolute levels of RNA and the corresponding protein exhibit a correlation coefficient of only 0.5 . However, there is a correlation between the sign of the change of RNA and protein (i.e., if an RNA exhibits increased expression, the corresponding protein exhibits increased expression). Therefore, the observed levels of RNA and protein are qualitatively similar but the actual magnitude of the changes are significantly different. The clear conclusion from these results is that the microarray and $2 \mathrm{D}$ protein gel data cannot be interpreted quantitatively. Furthermore, extensive control experiments indicate that poor reproducibility of the $2 \mathrm{D}$ protein gels compared to microarray hybridization is a major limiting factor for comparing transcription and translation results.

The effect of novobiocin and ciprofloxacin on $H$. influenzae gene expression was assessed at the minimum concentration of antibiotic known to inhibit bacterial growth (MIC) as well as at a tenfold higher concentration. Treatment with low concentrations of novobiocin resulted in only a few changes in gene expression, including increased expression of DNA gyrase subunit B and decreased expression of topoisomerase I, ribosome releasing factor as well as two hypothetical proteins. The changes in expression of DNA gyrase and topoisomerase I can be rationalized as a cellular response to the change in supercoiling due to inhibition of gyrase. It will be of interest to determine the function of the hypothetical proteins. Their grouping with gyrase and topoisomerase I suggests these hypothetical proteins may be involved in nucleic acid metabolism or DNA repair. If so, they may represent new targets for antibiotics that disrupt nucleic acid metabolism.

At high concentrations of novobiocin, $\sim 140$ genes exhibit significant changes in expression levels based on the microarray data. It is not obvious why, at the high concentration, such a large number of genes exhibit changes in expression. Perhaps the changes are secondary transcriptional effects due to the global changes in supercoiling. At both high and low concentrations, the length of time of exposure to novobiocin did not substantially affect the expression profile in that expression changes occurred at the earliest time points and remained relatively constant.

Exposure of $H$. influenzae to a low concentration of ciprofloxacin also results in expression changes of only a small number of genes. However, these genes are dominated by DNA repair proteins involved in the SOS response such as the recA, uvrA and lexA genes. This result is not surprising in that the drug causes DNA damage. Also, similar to the case with novobiocin, the expression levels of a large number of genes were altered at high ciprofloxacin concentrations, which could be secondary effects 
due to DNA damage and the impending death of the cell. It should be noted that ciprofloxacin is a bacteriocidal antibiotic for $H$. influenzae while novobiocin is bacteriostatic. Thus, treatment with high concentrations of ciprofloxacin will result in cell death while high concentrations of novobiocin will simply stop growth. This may result in a different cellular response and could explain why changes in gene expression continue to occur with prolonged exposure to ciprofloxacin, while cells exposed to novobiocin quickly exhibit a response but the response does not change upon prolonged exposure.

A common theme of both the novobiocin and ciprofloxacin data is that use of the low antibiotic concentrations for short periods of time provides the most interpretable results. Use of high concentrations for extended periods of time changes the expression of a large set of genes and these effects may be secondary to the action of the drug. Nevertheless, the changes that occur at high antibiotic concentrations are largely unique to each drug and thus provide a 'signature' for that drug. The demonstration that two antibiotics that act on the same bacterial target but by a different mechanism results in different gene expression profiles is an important contribution of this work. As the authors point out, having a set of signatures for a large number of antibiotics may be a very useful tool for understanding the mechanism of action of novel pharmaceuticals. It will be of great interest to determine if exposure of bacteria to drugs that have the same mechanism of action results in the same signature of gene expression. For example, does exposure to two different quinolones result in a similar pattern of gene expression? If these types of experiments are to be used to determine the mechanism of action of new antibiotics, the answer should be yes.

Can the cellular response to antibiotic exposure also be used to predict how resistance to the antibiotic will evolve? In the case of novobiocin and ciprofloxacin a common response is the induction of DNA gyrase. The major pathway of resistance to DNA gyrase inhibitors in clinical isolates is chromosomal mutations in the genes encoding gyrase (Davies 1994). Thus, the genes identified in the primary response to an antibiotic may be an indicator of genes involved in resistance. Using this strategy, the induced genes identified by microarrays could be targeted for DNA sequencing in resistant clinical isolates to identify mutations associated with drug resistance. However, if a large number of genes are induced, such as observed for the gyrase inhibitors at high drug concentrations, it would be very difficult to implement such a strategy.

Another mechanism of bacterial resistance to quinolones is efflux of the drug out of the cell (Poole 2000). H. influenzae is known to contain a homolog of the Escherichia coli acrAB multidrug efflux pump which can efflux novobiocin and ciprofloxacin from the cell (Sanchez et al. 1997). However, the expression of the $a c r A B$ pump does not appear to be affected by exposure to novobiocin or ciprofloxacin. Thus, there is not a correlation between exposure to gyrase inhibitors and expression of known efflux pump homologs. The role of efflux pumps in resistance to gyrase inhibitors in clinical isolates of $H$. influenzae is unknown.

Antibiotic-induced gene expression in Mycobacterium tuberculosis has previously been studied using microarray hybridization (Wilson et al. 1999). In these experiments, M. tuberculosis was exposed to isoniazid (INH), a drug that blocks the mycolic acid biosynthesis pathway in this organism. The response was similar to that observed in $H$. influenzae with low concentrations of gyrase inhibitors in that only 14 genes were induced. The induced genes are mainly involved in lipid metabolism. Therefore, the signature of induced expression is consistent with a block in M. tuberculosis lipid biosynthesis and is indicative of a drug that inhibits lipid metabolism. In addition, four of the induced genes are of unknown function. These genes may be involved in lipid metabolism and, if so, may represent targets for new antibiotics.

Taken together, the results of the $M$. tuberculosis and $H$. influenzae studies demonstrate that exposure of bacteria to antibiotics results in unique signatures of gene expression. These signatures are powerful tools for determining the mechanism of action of new antibiotics. In addition, determination of the function of the unknown genes that are induced in the primary response to antibiotic exposure may uncover potential new targets for antimicrobial development. Therefore, these technologies will facilitate the urgent search for new classes of antibiotics.

\section{REFERENCES}

Davies, J. 1994. Science 264: 375-382.

Gmuender, H., Kuratli, K., Di Padova, K., Gray, C.P., Keck, W., and Evers, S. 2001. Genome Res. 11: $28-42$

Piddock, L.J.V., Walters, R.N., and Diver, J.M. 1990. Antimicrob. Agents Chemother. 34: 2331-2336.

Poole, K. 2000. Antimicrob. Agents Chemother. 44: 2233-2241.

Sanchez, L., Pan, W., Vinas, M., and Nikaido, H. 1997. J. Bacteriol. 179: 6855-6857.

Wilson, M., DeRisi, J., Kristensen, H.-H., Imboden P., Rane, S., Brown, P.O., and Schoolnik, G.K. 1999. Proc. Natl. Acad. Sci. 96: 12833-12838. 


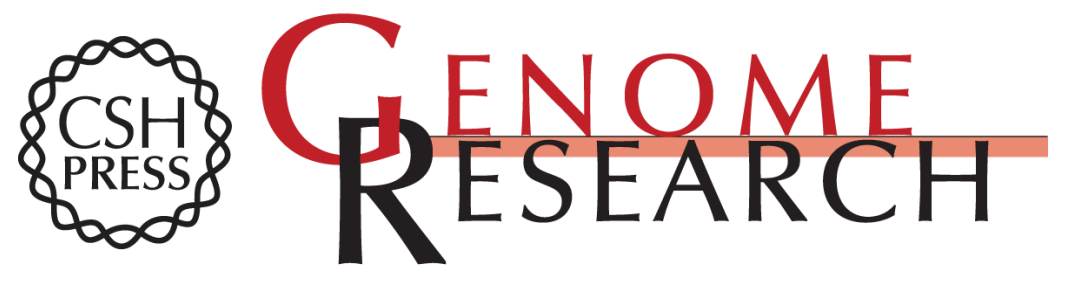

\section{Impending Doom: Antibiotic Exposure and Bacterial Gene Expression}

Timothy Palzkill

Genome Res. 2001 11: 1-2

Access the most recent version at doi:10.1101/gr.170901

References This article cites 6 articles, 6 of which can be accessed free at:

http://genome.cshlp.org/content/11/1/1.full.html\#ref-list-1

\section{License}

Email Alerting Receive free email alerts when new articles cite this article - sign up in the box at the Service top right corner of the article or click here.

\section{Affordable, Accurate Sequencing.}

To subscribe to Genome Research go to: https://genome.cshlp.org/subscriptions 